\title{
SUR LA FORME HERMITIENNE CANONIQUE DES ESPACES HOMOGENES COMPLEXES
}

\author{
J. L. KOSZUL
}

Introduction. Soit $G$ un groupe de Lie connexe et soit $B$ un sous-groupe fermé de $G$. Supposons que la variété homogène $G / B$ ou $G$ opère transitivement à gauche soit munie d'une structure complexe invariante par les opérations de $G$, et qu'il existe sur $G / B$ un volume invariant par $G$. Ce volume étant unique à un facteur constant près, la forme hermitienne de $G / B$ qui est canoniquement associée au volume et à la structure complexe se trouve entièrement déterminée par la structure complexe de $G / B$ et le groupe transitif $G$ (5). C'est cette forme hermitienne que nous appelons la forme hermitienne canonique de l'espace homogène complexe $G / B$. L'intérêt que présente cette forme pour la détermination des domaines bornés homogènes a été souligné par E. Cartan: une condition nécessaire pour que $G / B$ soit un domaine borné est en effet que cette forme soit définie positive (1).

Notre premier but est ici de calculer cette forme canonique à partir de données infinitésimales qui sont: l'algèbre de Lie $\mathfrak{g}$ de $G$, la sous-algèbre de $\mathfrak{g}$ qui correspond à $B$ et un endomorphisme de $\mathfrak{g}$ définissant la structure complexe invariante de $G / B$. Les paragraphes 1,2 , et 3 contiennent des préliminaires à ce calcul qui est achevé au $\$ 4$. La formule obtenue met en évidence le rôle joué par une certaîne forme invariante de degré 1 sur $G$ qui est definie par la structure complexe de $G / B$. Au $\S 5$, on en déduit des conditions nécessaires pour que la forme hermitienne de $G / B$ soit non dégénérée. Une étude détaillée du cas où $G$ est semi-simple et $B$ compact fait l'objet du $\$ 6$. Elle montre que des relations étroites existent dans ce cas entre la compacité de $G / B$ et les carrés négatifs de la forme canonique. Les résultats obtenus prouvent que les domaines bornés homogènes dont le groupe des automorphismes est semisimple sont des domaines bornés symétriques au sens de E. Cartan (3). Les problèmes que nous envisageons ici du point de vue de la "Théorie des groupes de Lie" se rattachent à un grand nombre de résultats récents de Géométrie différentielle $(2 ; 12)$.

1. Champs de vecteurs projetables. Soit $V$ une variété différentiable de classe $\infty$ et soit $F$ l'algèbre sur le corps des nombres réels des fonctions à valeurs réelles indéfiniment différentiables sur $V$. On notera $C$ le $F$-module des champs de vecteurs ${ }^{1}$ sur $V$ et $T(C)$ l'algèbre tensorielle du $F$-module $C$. Les éléments de degré $p$ de $T(C)$ constituent le $F$-module $T_{0}{ }^{p}$ des champs de

Reçu le 1 décembre, 1954.

${ }^{1}$ Dans tout ce mémoire, on ne considère que des variétés et des champs de tenseurs indéfiniment différentiables. 
tenseurs de type $(0, p)$ sur $V$. Le $F$-module $T_{p}^{q}=\operatorname{Hom}_{F}\left(T_{0}^{p}, T_{0}^{q}\right)$ est le module des champs de tenseurs de type $(p, q)$ sur $V$. Il y aura parfois avantage à considérer un élément $t \in T_{p}{ }^{q}$ comme une fonction $F$-linéaire de $p$ champs de vecteurs $X_{j}(j=1,2, \ldots, p)$ à valeurs dans $T_{0}{ }^{q}$; on écrira alors cette fonction $t\left(X_{1}, \ldots, X_{p}\right)$. Par abus de langage, les champs de tenseurs sur $V$ seront appelés des tenseurs de $V$.

Pour tout champ de vecteurs $X$, on notera $\theta(X)$ la transformation infinitésimale associée à $X$. Elle transforme un tenseur $t$ de type $(p, q)$ en un tenseur $\theta(X) . t$ de même type. On a

$$
\begin{aligned}
& (\theta(X) . t)\left(X_{1}, \ldots, X_{p}\right) \\
& \quad=\theta(X) \cdot\left(t\left(X_{1}, \ldots, X_{p}\right)\right)-\sum_{j=1}^{p} t\left(X_{1}, \ldots,\left[X, X_{j}\right], \ldots, X_{p}\right)
\end{aligned}
$$

quels que soient les champs $X_{j} \in C(j=1,2, \ldots, p)$.

Soit maintenant $\pi$ une application différentiable de $V$ sur une variété différentiable $W$. A tout tenseur $t$ de type $(p, 0)$ sur $W$, l'application $\pi$ fait correspondre un tenseur de même type sur $V$ noté $\pi^{*} . t$. L'application $\pi^{*}$ est un isomorphisme. Soit $F^{\prime} \subset F$ l'image par $\pi^{*}$ l'algèbre des fonctions différentiables de $W$. Un champ de vecteurs $X$ sur $V$ sera dit projetable si $\theta(X)$. $F^{\prime} \subset F^{\prime}$. Si $X$ est projetable, il existe sur $W$ un champ de vecteurs $X^{\prime}$ et un seul tel que $\theta(X) \pi^{*} . f=\pi^{*} \theta\left(X^{\prime}\right) . f$ pour toute fonction différentiable $f$ sur $W$. Ce champ $X^{\prime}$ sera appelé la projection de $X$ et sera noté $\pi$.X. L'application $\pi$ vérifie les propriétés suivantes:

(1.2) si $X$ et $Y$ sont projetables, alors $X+Y$ et $[X, Y]$ sont projetables et $\pi \cdot(X+Y)=\pi . X+\pi \cdot Y, \pi \cdot[X, Y]=[\pi \cdot X, \pi \cdot Y]$,

(1.3) si $X$ est projetable et si $f$ est une fonction différentiable sur $W$, alors $\left(\pi^{*} . f\right) X$ est projetable et $\pi .\left(\left(\pi^{*} . f\right) X\right)=f(\pi . X)$,

(1.4) si $t$ est un tenseur de type $(p, 0)$ sur $W$ et si les $X_{j}(j=1,2, \ldots, p)$ sont des champs de vecteurs projetables sur $V$, on a

$$
\left(\pi^{*} . t\right)\left(X_{1}, \ldots, X_{p}\right)=\pi^{*} .\left(t\left(\pi . X_{1}, \ldots, \pi \cdot X_{p}\right)\right),
$$

(1.5) pour tout tenseur $t$ de type $(p, 0)$ de $W$ et tout champ de vecteurs projetable $X$ sur $V, \theta(X) \pi^{*} . t=\pi^{*} \theta(\pi . X) . t$.

Si $V$ est un espace fibré différentiable localement trivial et si $\pi: V \rightarrow W$ est la projection de $V$ sur sa base, alors tout champ de vecteurs sur $W$ est projection d'un champ de vecteurs sur $V$.

Par la suite, on utilisera ces champs projetables dans le cas où $V$ est un groupe de Lie connexe $G$ et $W$ un espace homogène de groupe $G$. Plus précisément, un sous-groupe fermé $B$ sera donné ${ }^{2}$ dans $G$ et $W=G / B$ sera la variété quotient de $G$ par la relation d'équivalence: $s \backsim s^{\prime}$ lorsque $s^{\prime} s^{-1} \in B$. La projection $\pi: G \rightarrow G / B$ associe à chaque $s \in G$ sa classe d'équivalence consid-

${ }^{2}$ Le sous-groupe $B$ ne sera pas supposé connexe. 
érée comme élément de $G / B$. On désignera par g l'algèbre de Lie des champs de vecteurs invariants à gauche sur $G$ et par b la sous-algèbre des $X \in \mathfrak{g}$ qui, au point neutre $e$ de $G$, sont tangents à $B$. Cette sous-algèbre $\mathfrak{b}$ est canoniquement isomorphe à l'algèbre de Lie des champs de vecteurs invariants à gauche sur $B$. La translation à droite par un élément $s \in G$ transforme tout champ de vecteurs $X$ sur $G$ en un champ noté $X s$. Pour qu'un champ de vecteurs $X$ sur $G$ soit projetable sur $G / B$, il faut et il suffit que, pour tout $s \in B$, le champ. de vecteurs $X s-X$ soit dans le $F$-module $F \mathfrak{b}$ engendré par les éléments de $\mathfrak{b}$. Les champs de vecteurs dont la projection est nulle sont les éléments de $F \mathfrak{b}$. En particulier, les champs de vecteurs invariants à droite sur $G$ sont projetables. Les transformations infinitésimales associées à leurs projections correspondent aux opérations de $G$ opérant à gauche sur $G / B$.

Soit $T\left(e^{\prime}\right)$ l'espace vectoriel des vecteurs tangents à $G / B$ au point $e^{\prime}=\pi . e$. Les opérations de $G$ sur $G / B$ définissent une représentation linéaire de $B$ dans $T\left(e^{\prime}\right)$ qui sera notée à gauche. Les $X \in \mathfrak{g}$ ne sont pas projetables en général, mais en associant à tout $X \in \mathfrak{g}$ l'image par l'application linéaire tangente à $\pi$ du vecteur $X_{e}$ défini par $X$ au point $e$, on obtient une application linéaire $\pi^{\prime}$ de $\mathfrak{g}$ sur $T\left(e^{\prime}\right)$ dont le noyau est $\mathfrak{b}$. Pour tout $s \in B$, on a alors $s\left(\pi^{\prime} . X\right)=\pi^{\prime} .\left(X s^{-1}\right)$ quel que soit $X \in \mathfrak{g}$.

2. Structure complexe invariante sur un espace homogène. Les notations restant celles du paragraphe précédent, supposons que la variété $G / B$ soit munie d'une structure presque complexe invariante par les opérations de $G$. Cette structure est définie par un tenseur $I$ de type $(1,1)$ sur $G / B$, c'est à dire un endomorphisme du module des champs de vecteurs sur $G / B$, qui est invariant par $G$ et vérifie la condition $I^{2} \cdot U=-U$ pour tout champ de vecteurs $U$. Soit $I_{e}$, l'endomorphisme de $T\left(e^{\prime}\right)$ défini par $I$ au point $e^{\prime}$. De l'invariance de $I$ par $G$ résulte que $I_{e}$, commute avec les opérations de $B$ dans. $T\left(e^{\prime}\right)$. Soit $J$ un endomorphisme de l'espace $\mathfrak{g}$ tel que

$$
\begin{aligned}
\pi^{\prime} J & =I_{e}, \pi^{\prime}, \\
J . \mathfrak{b} & =(0) .
\end{aligned}
$$

Des propriétés de $I_{e}$, résultent que

$$
J^{2} . X=-X(\bmod \mathfrak{b}) \text { pour tout } X \in \mathfrak{g}
$$

Réciproquement, soit $J$ un endomorphisme de $\mathfrak{g}$ vérifiant les conditions: (2.2), (2.3) et (2.4). Il existe un endomorphisme $I_{e}$, de $T\left(e^{\prime}\right)$ et un seul qui vérifie la condition (2.1). Cet endomorphisme est de carré -1 et commute avec les opérations de $B$. Il définit donc un tenseur $I$ de type $(1,1)$ sur $G / B$ invariant par $G$ et tel que $I^{2} . U=-U$ pour tout champ de vecteurs $U$ sur. $G / B$, c'est à dire une structure presque complexe sur $G / B$.

Le $F$-module des champs de vecteurs sur $G$ étant un module libre qui admet pour base une base de l'espace $\mathfrak{g}$, l'endomorphisme $J$ de $\mathfrak{g}$ est la restric-. 
tion d'un tenseur de type $(1,1)$ sur $G$ qui est invariant par les translations à gauche et que l'on notera encore $J$. D'après $(2.4), J .(X s)-(J . X) s \in F \mathfrak{b}$ pour tout $s \in G$ et comme d'après $(2.2) J .(F \mathfrak{b})=(0)$, on voit que si $X$ est un champ de vecteurs projetable sur $G$, alors $J . X$ est également projetable. De plus, $J$ et $I$ étant invariant par $G$, la condition (2.1) entraîne que l'on a alors

$$
\pi .(J \cdot X)=I .(\pi \cdot X) .
$$

Supposons maintenant que la structure presque complexe donnée sur $G / B$ soit une structure complexe. Le tenseur $R$ de type $(2,1)$ sur $G / B$ défini par

$$
R(U, V)=[U, V]+I .[I . U, V]+I .[U, I . V]-[I . U, I . V]
$$

où $U$ et $V$ sont des champs de vecteurs sur $G / B$ est alors (8) égal à 0 . Associons comme précédemment à $I$ un tenseur $J$ sur $G$ et posons

$$
S(X, Y)=[X, Y]+J .[J . X, Y]+J .[X, J Y]-[J . X, J . Y]
$$

quels que soient les champs de vecteurs $X, Y$ sur $G$. Si $X$ et $Y$ sont des champs invariants à droite sur $G$, donc projetables, alors $S(X, Y)$ est projetable et $\pi . S(X, Y)=R(\pi . X, \pi . Y)=0$ d'après (1.2) et (2.5). Par conséquent, le champ de vecteurs $S(X, Y)$ est dans $F \mathfrak{b}$. Nous allons voir que ceci est encore vérifié pour des champs de vecteurs $X, Y$ arbitraires sur $G$. Il suffit pour cela d'observer que, pour toute fonction $f \in F$, on a

$$
S(X, f Y)=f S(X, Y)+(\theta(x) \cdot f)\left(Y+J^{2} \cdot Y\right)
$$

quels que soient les champs ${ }^{3}$ de vecteurs $X, Y$. D'après (2.3), $Y+J^{2} . Y \in F \mathfrak{b}$, par suite $S(X, f Y)=f S(X, Y) \bmod F \mathfrak{b}$. Comme $S(X, Y)=-S(Y, X)$ et que le $F$-module des champs de vecteurs sur $G$ est engendré par les champs invariants à droite, ceci prouve que la relation $S(X, Y) \in F \mathfrak{b}$ est vérifiée quels que soient $X$ et $Y$. En particulier, si $X, Y \in \mathfrak{g}, S(X, Y) \in F \mathfrak{b} \cap \mathfrak{b}=\mathfrak{b}$. On voit donc que, si $I$ définit sur $G / B$ une structure complexe, alors l'endomorphisme $J$ de $\mathfrak{g}$ vérifie la condition

$$
[X, Y]+J .[J . X, Y]+J .[X, J . Y]-[J . X, J . Y] \in \mathfrak{b}
$$

quels que soient ${ }^{4} X, Y \in \mathfrak{g}$.

Réciproquement, si l'on part d'un endomorphisme $J$ de $\mathfrak{g}$ qui vérifie les conditions (2.2), (2.3), (2.4) et (2.6), la structure presque complexe sur $G / B$ qui lui correspond est une structure complexe invariante par $G$. En effet, $S(X, Y) \in F \mathfrak{b}$ quels que soient les champs de vecteurs $X, Y$ sur $G$ et par suite, si $X$ et $Y$ sont projetables, $R(\pi . X, \pi . Y)=0$. Tout champ de vecteurs sur

${ }^{3}$ D'après la formule $[X, f Y]=f[X, Y]+(\theta(X) . f) Y$, valable quels que soient les champs de vecteurs $X, Y$ et la fonction $f$.

${ }^{4}$ Des conditions $(2.2)$ et $(2.3)$ résulte que $J\left(J^{2}+1\right)=0$. On vérifie facilement que (2.6) équivaut à la condition suivante: $\operatorname{si} J^{C}$ est le prolongement de $J$ à l'algèbre $\mathfrak{g}^{C}$ complexifiée de $g$, le sous-espace des zéros de $J^{C}\left(J^{C}-i\right)$ est une sous-algèbre de $\mathfrak{g}^{C}$. 
$G / B$ étant projection d'un champ de $G$, on a $R=0$ c'est à dire que le tenseur $I$ vérifie la condition d'intégrabilité. Comme $I$ est invariant par $G$, il est analytique réel pour la structure analytique réelle canonique de $G / B$. On sait que dans ces conditions, $I$ définit sur $G / B$ une structure complexe (8).

3. Une expression de la forme hermitienne associée à un volume. Nous supposerons ici que $V$ est une variété analytique complexe de dimension réelle $2 n$ sur laquelle est donnée une forme différentielle réelle $\omega$ de degré $2 n$ partout de rang $2 n$ sur $V$. Un champ de vecteurs complexes sur $V$ est une combinaison $X+(-i)^{\frac{1}{2}} Y$ où $X$ et $Y$ sont des champs de vecteurs réels. Pour tout champ de vecteurs complexes $\mathfrak{X}$ sur $V$, on notera $\partial . \mathfrak{X}$ la divergence de $\mathfrak{X}$, c'est à dire la fonction différentiable à valeurs complexes définie par $\theta(\mathfrak{X}) \cdot \omega=(\partial . \mathfrak{X}) \omega$. On vérifie facilement les formules

$$
\theta(\mathfrak{X}) \partial . \mathfrak{Y}-\theta(\mathfrak{Y}) \partial . \mathfrak{X}=\partial .[\mathfrak{X}, \mathfrak{Y}], \quad \partial .(f \mathfrak{X})=f(\partial . \mathfrak{X})+\theta(\mathfrak{X}) . f,
$$

qui sont valables quels que soient les champs $\mathfrak{X}, \mathfrak{Y}$ et quelle que soit la fonction complexe $f$.

Soient $z_{j}(j=1,2, \ldots, n)$ des coordonnées locales complexes au voisinage d'un point de $V$ et soient $Z_{i}$ les champs de vecteurs complexes tels que $\theta\left(3_{j}\right) \cdot f=\partial f / \partial z_{j}$ pour toute fonction différentiable à valeurs complexes définie au voisinage de ce point. Le volume $\omega$ s'écrit localement

$$
\omega=K d z_{1} \wedge \ldots d z_{n} \wedge d \bar{z}_{1} \wedge \ldots d \bar{z}_{n}
$$

où $K$ est une fonction différentiable à valeurs réelles ou imaginaires pures suivant la parité de $n$. On a, pour tout $i$,

$$
\partial . \AA_{j}=\frac{\partial \log K}{\partial z_{j}}, \quad \quad \partial \cdot \overline{\grave{z}}_{j}=\frac{\partial \log K}{\partial \bar{z}_{j}} .
$$

Soit $\mathfrak{Y}$ un champ de vecteurs analytique sur $V$ qui s'écrira localement

$$
\mathfrak{Y}=\sum_{j=1}^{n} b_{j} \mathfrak{Z}_{j}
$$

où les $b_{i}$ sont des fonctions analytiques et soit $\mathfrak{X}$ un champ de vecteurs de type analytique sur $V$, c'est à dire s'écrivant localement $\mathfrak{X}=\sum a_{j} \bigcap_{j}$ où les $a_{j}$ sont des fonctions différentiables à valeurs complexes. D'après (3.1), on a

$$
\partial \cdot \overline{\mathfrak{Y}}=\sum_{j=1}^{n} \frac{\partial \bar{b}_{j}}{\partial \bar{z}_{j}}+\sum_{k=1}^{n} \frac{\partial \log K}{\partial \bar{z}_{k}} \bar{b}_{k} .
$$

Puisque $\partial \bar{b}_{j} / \partial z_{k}=0$ quels que soient $j$ et $k$, on a donc

$$
\theta(\mathfrak{X}) \cdot(\partial \cdot \overline{\mathfrak{Y}})=\sum_{j, k=1}^{n} \frac{\partial^{2} \log K}{\partial z_{j} \partial \bar{z}_{k}} a_{j} \bar{b}_{k} .
$$

Or le tenseur dont les composantes sont localement

$$
\frac{\partial^{2} \log K}{\partial z_{j} \partial \bar{z}_{k}}
$$


est la forme hermitienne (1) associé au volume $\omega$. On voit donc que (3.2) Si $\mathfrak{Y}$ est un champ analytique et si $X$ est un champ de type analytique, alors $\theta(\mathfrak{X}) \cdot(\partial \cdot \overline{\mathfrak{Y}})=h(\mathfrak{X}, \overline{\mathfrak{Y}})$ où $h$ désigne la forme hermitienne associée au volume $\omega$.

On observera que cette expression de $h$, dont le caractère intrinsèque nous sera commode dans la suite, cesse d'être valable pour des champs $\mathfrak{X}, \mathfrak{Y}$ quelconques.

La restriction de $h$ aux champs de vecteurs réels est une forme bilinéaire symétrique réelle qui vérifie la condition $h(I . X, Y)+h(X, I . Y)=0$, où $I$ est le tenseur de type $(1,1)$ sur $V$ qui définit la structure complexe. On désignera par $a$ la forme antisymétrique réelle $a(X, Y)=h(X, I Y)$. Si $X$ et $Y$ sont deux champs de vecteurs réels sur $V$ et si $\mathfrak{X}=X-i I . X$ et $\mathfrak{Y}=Y-i I . Y$ sont les champs de type analytique qui leur correspondent, on a $4 a(X, Y)=$ $i h(\mathfrak{X}+\overline{\mathfrak{X}}, \mathfrak{Y}-\overline{\mathfrak{Y}})=i(h(\overline{\mathfrak{X}}, \mathfrak{Y})-h(\mathfrak{X}, \overline{\mathfrak{Y}}))$. Si $Y$ est conforme, c'est à dire si $\mathfrak{Y}$ est analytique, la formule (3.2) montre donc que

$$
2 a(X, Y)=\theta(X) .(\partial I . Y)-\theta(I . X) .(\partial . Y) .
$$

Compte tenu de (3.1), il en résulte que:

(3.3) Si $X$ et $Y$ sont deux champs de vecteurs conformes tels que $\partial . X=0$ et ว. $Y=0$, alors $2 a(X, Y)=\partial .(I[X, Y])$.

4. Calcul de la forme hermitienne canonique des espaces homogènes complexes. Les notations étant celles du $\$ 1$, supposons qu'il existe sur $G / B$ un volume $\omega$ invariant par $G$. Une condition nécessaire et suffisante pour qu'il en soit ainsi est que la représentation linéaire de $B$ dans $T\left(e^{\prime}\right)$ se fasse par des endomorphismes de déterminant 1 . Le volume invariant, s'il existe, est déterminé à un facteur constant près. Etant donné un endomorphisme $\gamma$ de l'espace $\mathfrak{g}$ tel que $\gamma \cdot \mathfrak{b} \subset \mathfrak{b}$, on notera $\operatorname{Tr}_{\mathfrak{g} / \mathfrak{b}} \gamma$ la trace de l'endomorphisme de $\mathfrak{g} / \mathfrak{b}$ déduit de $\gamma$ par passage au quotient. L'existence d'un volume invariant sur $G / B$ implique $\operatorname{Tr}_{\mathfrak{g}} / \mathfrak{b}$ ad $(X)=0$ pour tout ${ }^{5} X \in \mathfrak{b}$.

Soit $X_{j}(j=1,2, \ldots m)$ une base de l'espace $\mathrm{g}$ telle que les $X_{j}$ dont l'indice vérifie $2 n<j \leqslant m$ constituent une base de $\mathfrak{b}$, les entiers $m$ et $2 n$ étant respectivement les dimensions de $G$ et $G / B$. Soient $\xi_{j}(j=1,2, \ldots m)$ les formes de degré 1 invariantes à gauche sur $G$ définies par $\xi_{j}\left(X_{k}\right)=\delta_{j k}$ (indice de Kronecker). L'image inverse $\Omega=\pi^{*} . \omega$ de la forme $\omega$ est égale, à un facteur constant près, à $\xi_{1} \wedge \xi_{2} \wedge \ldots \xi_{2 n}$. On pourra supposer la base $X_{f}$ choisie de sorte que ce facteur soit égal à 1 .

Lemme 1. Pour tout champ de vecteurs projetable $X$ sur $G$, on a

$$
\pi^{*} \partial \pi \cdot X=\sum_{j=1}^{2 n} \xi_{j}\left(\left[X_{j}, X\right]\right) .
$$

\footnotetext{
${ }^{5} \mathrm{Si} B$ est connexe, cette condition $\operatorname{Tr} \mathfrak{g} / \mathfrak{b} a d(X)=0$ pour $X \in \mathfrak{b}$ équivaut à l'existence d'un volume invariant.
} 
En effet, d'après (1.5),

$$
\left(\pi^{*} \partial \pi . X\right) \Omega=\pi^{*} .((\partial \pi . X) \omega)=\pi^{*} .(\theta(\pi . X) . \omega)=\theta(X) \pi^{*} . \omega=\theta(X) . \Omega .
$$

D'autre part,

$$
\begin{aligned}
\theta(X) . \Omega & =\sum_{j=1}^{2 n} \xi_{1} \wedge \ldots\left(\theta(X) . \xi_{j}\right) \wedge \ldots \xi_{2 n} \\
& =\sum_{j=1}^{2 n} \sum_{k=1}^{m}\left(\theta(X) . \xi_{j}\right)\left(X_{k}\right) \xi_{1} \wedge \ldots \xi_{j-1} \wedge \xi_{k} \wedge \xi_{j+1} \wedge \ldots \xi_{2 n} .
\end{aligned}
$$

Puisque les fonctions $\xi_{j}\left(X_{k}\right)$ sont des constantes, la formule (1.1) montre que

$$
\left(\theta(X) \cdot \xi_{j}\right)\left(X_{k}\right)-\xi_{j}\left(\left[X, X_{k}\right]\right)=\theta(X) \cdot\left(\xi_{j}\left(X_{k}\right)\right)=0 .
$$

Or, pour $2 n<k \leqslant m, X_{k} \in \mathfrak{b}$, donc d'après (1.2), $\pi \cdot\left[X, X_{k}\right]=0$, c'est à dire que $\left[X, X_{k}\right]$ est dans le module $F \mathfrak{b}$ engendré par $\mathfrak{b}$. Il en résulte que $\xi_{j}\left(\left[X, X_{k}\right]\right)=0$ pour tout $i>2 n$ et par suite,

$$
\theta(X) . \Omega=\left(\sum_{j=1}^{2 n} \xi_{j}\left(\left[X_{j}, X\right]\right)\right) \Omega .
$$

La formule (4.1) en résulte parceque $\Omega \neq 0$ en tout point de $G$.

Supposons maintenant donnée sur $G / B$ une structure complexe invariante par $G$. Cette structure sera définie par un endomorphisme $J$ de l'espace $\mathfrak{g}$ vérifiant les propriétés (2.2), (2.3), (2.4) et (2.6). La restriction aux champs de vecteurs réels de la forme hermitienne canonique $h$ de $G / B$ a pour image inverse sur $G$ une forme bilinéaire symétrique invariante à gauche $\eta=\pi^{*} . h$. De même, la forme antisymétrique a définie par $a(U, V)=h(U, I . V)$ aura pour image inverse une forme antisymétrique $\alpha=\pi *^{*} a$ invariante à gauche sur $G$. Il résulte de $(1.4)$ et (2.5) que

$$
\eta(X, Y)=\alpha(J . X, Y),
$$

quels que soient les champs de vecteurs $X, Y$ sur $G$. Nous allons procéder au calcul de $\alpha$.

Soit $X$ un champ de vecteurs invariant à droite sur $G$. A sa projection $\pi . X$ est associée une transformation infinitésimale du groupe $G$ opérant sur $G / B$ Comme ces opérations de $G$ conservent le volume $\omega$ et la structure complexe de $G / B, \pi . X$ est un champ de vecteurs conforme et $\partial \pi . X=0$. Si donc $X$ et $Y$ sont deux champs de vecteurs invariants à droite sur $G$, on a d'après (3.3), (1.2) et (2.5):

$$
2 a(\pi . X, \pi . Y)=\partial .(I .[\pi . X, \pi . Y])=\partial .(I \pi \cdot[X, Y])=\partial .(\pi J .[X, Y]) .
$$

D'après (1.4) et (4.1), on a donc

$$
2 \alpha(X, Y)=\sum_{j=1}^{2 n} \xi_{j}\left(\left[X_{j}, J .[X, Y]\right]\right) .
$$

De cette formule qui donne $\alpha(X, Y)$ lorsque $X$ et $Y$ sont des champs invariants à droite, il nous reste à déduire la valeur de $\alpha(X, Y)$ lorsque $X$ et $Y$ 
sont des champs invariants à gauche sur $G$. Soient $X^{\prime}$ et $Y^{\prime}$ les champs invariants à droite qui coïncident respectivement au point neutre $e$ de $G$ avec les champs invariants à gauche $X$ et $Y$. Posons

$$
\left[X^{\prime}, Y^{\prime}\right]=\sum_{k=1}^{m} f_{k} X_{k}=T
$$

où les $f_{k}$ sont des fonctions différentiables sur $G$. Comme $T$ est invariant à droite, on $\mathrm{a}^{3}$ pour tout indice $j$ :

$$
0=\left[X_{j}, T\right]=\sum_{k=1}^{m}\left(\theta\left(X_{j}\right) \cdot f_{k}\right) X_{k}+\sum_{k=1}^{m} f_{k}\left[X_{j}, X_{k}\right]
$$

et par suite,

$$
\left[X_{j}, J . T\right]=\sum_{k=1}^{m}\left(\theta\left(X_{j}\right) \cdot f_{k}\right)\left(J . X_{k}\right)+\sum_{k=1}^{m} f_{k}\left[X_{j}, J . X_{k}\right]
$$

Or le champ invariant à gauche

$$
\sum_{k=1}^{m} f_{k}(e) X_{k}
$$

qui coïncide en $e$ avec $\left[X^{\prime}, Y^{\prime}\right]$ est égal à $-[X, Y]$. Par conséquent, au point $e,\left[X_{j}, J . T\right]$ coïncide avec le champ invariant à gauche $J .\left[X_{j},[X, Y]\right]-$ $\left[X_{j}, J .[X, Y]\right]$. La formule (4.3) donne donc comme valeur de la fonction $\alpha(X, Y)$ au point $e$ :

$$
2 \alpha(X, Y)=\sum_{j=1}^{2 n} \xi_{j}\left(\left[J .[X, Y], X_{j}\right]-J .\left[[X, Y], X_{j}\right]\right)
$$

Comme $\alpha$ est invariante à gauche, cette égalité est vérifiée en tout point de $G$. Pour interpréter cette formule, on désignera par $\psi$ la forme de degré 1 invariante à gauche sur $G$ qui est définie par

$$
\psi(X)=\operatorname{Tr}_{\mathfrak{g} / \mathfrak{b}}(\operatorname{ad}(J . X)-J \operatorname{ad}(X))
$$

pour tout $X \in \mathfrak{g}$. Cette définition est justifiée car, d'après (2.4), si $Y \in \mathfrak{b}$, $J \operatorname{ad}(Y)-\operatorname{ad}(Y) J$ applique $\mathfrak{g}$ dans $\mathfrak{b}$ et par suite, l'endomorphisme ad $(J . X)-$ $J \operatorname{ad}(X)$ applique $\mathfrak{b}$ dans $\mathfrak{b}$ quel que soit $X \in \mathfrak{g}$. La formule (4.5) s'écrit alors,

$$
2 \alpha(X, Y)=\psi([X, Y])=(\delta . \psi)(X, Y)
$$

quels que soient $X, Y \in \mathfrak{g}$. D'où le

THÉORÈme 1. Si h est la forme hermitienne canonique de $G / B$, l'image inverse sur $G$ de la forme antisymétrique $h(U, I . V)$ est la différentielle extérieure de la forme invariante a gauche $\psi$ que définit (4.5).

Cette forme $\psi$ est déterminée sans ambiguité par la structure complexe de $G / B$. En effet, si $J^{\prime}$ est un autre endomorphisme de g qui définit la même structure complexe sur $G / B$, on a $J^{\prime} . X=J . X \bmod \mathfrak{b}$ pour tout $X \in \mathfrak{g}$ et par suite, 


$$
\begin{aligned}
\operatorname{Tr}_{\mathfrak{g} / \mathfrak{b}}\left(\operatorname{ad}\left(J^{\prime} . X\right)-J^{\prime} \operatorname{ad}(X)\right) & -\operatorname{Tr}_{\mathfrak{g} / \mathfrak{l}}(\operatorname{ad}(J . X)-J \operatorname{ad}(X)) \\
& =\operatorname{Tr}_{\mathfrak{g} / \mathfrak{l}}\left(\operatorname{ad}\left(J^{\prime} . X-J . X\right)-\left(J^{\prime}-J\right) \operatorname{ad}(X)\right)=0
\end{aligned}
$$

car $\operatorname{Tr}_{\mathfrak{g} / \mathfrak{b}} \operatorname{ad}(Y)=0$ pour tout $Y \in \mathfrak{b}$.

(4.7) La forme $\psi$ est invariante par les translations à droite par des éléments de $B$. Soit en effet $r(s)$ l'automorphisme $X \rightarrow X s$ de l'algèbre de Lie $g$ que définit la translation à droite par un élément $s \in G$. La condition (2.4) signifie que, si $t \in B$, alors $J r(t)-r(t) J$ applique $\mathfrak{g}$ dans $\mathfrak{b}$; par suite

$$
\operatorname{Tr}_{\mathfrak{g} / \mathfrak{b}}(\operatorname{ad}(J r(t) . X)-\operatorname{ad}(r(t) J . X))=0
$$

pour tout $X \in \mathfrak{g}$. Il en résulte par un calcul simple

$$
\psi(r(t) . X)=\operatorname{Tr}_{\mathfrak{g} / \mathfrak{b} r}(t)(\operatorname{ad}(J . X)-J \operatorname{ad}(X)) r(t)^{-1}=\psi(X),
$$

c'est à dire l'invariance de $\psi$ par la translation à droite par $t$.

5. Les espaces homogenes complexes dont la forme hermitienne canonique est non dégénérée. Soit $B^{\prime}$ le sous-groupe fermé des $t \in G$ tels que $\psi$ soit invariante par la translation à droite par $t$. Ce sous-groupe qui contient $B$ (4.7) est le sous-groupe de stabilité de $\psi$ dans la représentation duale de la représentation adjointe $s \rightarrow r(s)$. Les $X \in \mathfrak{g}$ qui, en $e$ sont tangents à $B^{\prime}$ constituent une sous-algèbre $\mathfrak{b}^{\prime}$ de $\mathfrak{g}$ contenant $\mathfrak{b}$. Pour que $X \in \mathfrak{b}^{\prime}$, il faut et il suffit que $\theta(X) . \psi=0$, autrement dit que $2 \alpha(X, Y)=\psi([X, Y])=0$ pour tout $Y \in \mathfrak{g}$. Ceci signifie que les $X \in \mathfrak{b}^{\prime}$ sont les éléments de $\mathfrak{g}$ dont l'image par $\pi^{\prime}$ (cf. §2) est un élément de $T\left(e^{\prime}\right)$ orthogonal à $T\left(e^{\prime}\right)$ pour la forme hermitienne canonique de $G / B$. On a donc le critère:

(5.1) Pour que la forme hermitienne canonique de $G / B$ soit non dégénérée, il faut et il suffit que $\mathfrak{b}^{\prime}=\mathfrak{b}$, c'est à dire que $B$ soit un sous-groupe ouvert $d u$ groupe de stabilité de $\psi$ dans la représentation duale de la représentation adjointe de $G$.

Si la forme canonique est non dégénérée, l'espace homogène $G / B$ est donc un revêtement de la trajectoire $G / B^{\prime}$ de $\psi$ dans cette représentation duale. ${ }^{6}$

Si l'on suppose $G$ semi-simple, la forme de Killing définit un isomorphisme entre la représentation adjointe et la représentation duale. De (5.1) résulte alors que

(5.2) Si G est semi-simple et si la forme hermitienne canonique de $G / B$ est non dégénérée, alors $B$ est un sous-groupe ouvert du centralisateur d'un sousgroupe à un paramètre connexe de G.

Si la forme $\psi$ est nulle sur $\mathfrak{b}$, alors, $\psi$ étant invariante à droite par $B$, il existe une forme $\phi$ de degré 1 sur $G / B$ telle que ${ }^{7} \pi^{*} . \phi=\psi$. Cette forme est invariante

\footnotetext{
${ }^{6} \mathrm{Si} B$ est discret, il doit donc exister une trajectoire ouverte dans la représentation duale de la représentation adjointe de $G$.

${ }^{7} \mathrm{La}$ forme $\psi$ est basique au sens de (7).
} 
par $G$ et, comme

$$
2 \pi^{*} . a=2 \alpha=\delta \psi=\delta \pi^{*} . \phi=\pi^{*} \delta . \phi,
$$

on a $\delta . \phi=2 a$. Si de plus la forme canonique de $G / B$ est non dégénérée, le volume invariant $\omega$ sur $G / B$ sera à un facteur constant près la puissance extérieure $n$-ième de $a$ donc la dérivée extérieure d'une forme invariante $\phi \wedge a \wedge a \ldots a$. On voit donc que

(5.3) Si le volume invariant sur $G / B$ n'est pas la différentielle extérieure d'une forme invariante de degré $2 n-1$ sur $G / B$, et si la forme hermitienne canonique de $G / B$ n'est pas dégénérée, alors $\psi$ n'est pas nulle sur $\mathfrak{b}$ et en particulier, $\mathfrak{b} \neq$ $[\mathfrak{b}, \mathfrak{b}]$.

On sait (11, Théorème 12.1) que si $G$ est unimodulaire et si la représentation adjointe de $B$ dans $g$ est complètement réductible, alors le volume invariant de $G / B$ n'est pas la différentielle d'une forme invariante. De (5.3) résulte donc que

(5.4) Si $G$ est unimodulaire, si B est compact et si la forme hermitienne canoni$q u e$ de $G / B$ est non dégénérée, alors le centre de $B$ est de dimension ${ }^{8}>0$.

Supposons maintenant que $G$ opère de manière effective sur $G / B$; le sousgroupe $B$ ne contient alors aucun sous-groupe distingué de $G$ autre que $(e)$. Si la forme hermitienne canonique de $G / B$ est non dégénérée, la composante connexe de l'élément neutre dans le centre de $G$ se réduit à $(e)$ car, d'après (5.1) elle est contenue dans $B$. Le centre de $G$ est donc un sous-groupe discret. D'autre part, la forme canonique étant toujours supposée non dégénérée, si la représentation linéaire de $B$ dans $\mathfrak{g} / \mathfrak{b}$ qui est définie par la représentation adjointe de $G$ est une représentation simple, alors $G$ est un groupe semi-simple. En effet, si $\mathfrak{m}$ est un idéal abélien de $\mathfrak{g}$, son image dans le quotient $\mathfrak{g} / \mathfrak{b}$ est soit (0) soit $\mathfrak{g} / \mathfrak{b}$. Dans le premier cas $\mathfrak{m} \subset \mathfrak{b}$ et donc $\mathfrak{m}=(0)$ puisque $G$ est effectif. Dans le second, la formule $2 \alpha(X, Y)=\psi([X, Y])$ montre que $\mathfrak{m} \cap \mathfrak{b}^{\prime}=\mathfrak{b}$, donc que $\mathfrak{g}=(0)$.

6. Le cas d'un groupe transitif semi-simple. Dans ce paragraphe, on supposera que $G$ est un groupe semi-simple et que le sous-groupe $B$ est un sous-groupe ouvert du centralisateur d'un sous-groupe à un paramètre $L$ de $G$. On supposera en outre que $B$ est compact. On se propose de montrer que $G / B$ possède des structures complexes invariantes et d'étudier les formes hermitiennes correspondantes.

Soit $M$ un sous-groupe compact maximal de $G$ contenant $B$, donc $L$. Puisque $M$ est connexe (10), le centralisateur de $L$ dans $M$ est un sous-groupe connexe (9) de $M$ et il en résulte que $B$ est connexe. Soit $Z$ le centre de $G$ et $G_{a}=G / Z$ le groupe adjoint de $g$. L'image canonique de $B$ dans $G / Z$ est un sous-groupe compact $B_{a}$ de $G_{a}$. Soit $K_{a}$ un sous-groupe compact maximal de $G_{a}$ contenant

\footnotetext{
${ }^{8} \mathrm{Ce}$ résultat est à rapprocher de certains résultats de Lichnerowicz (12).
} 
$B_{a}$ et soit $K$ le sous-groupe de $G$ image inverse de $K_{a}$ dans $G$. La composante connexe de l'élément neutre dans le centre de $K_{a}$ est un sous-groupe fermé de $B$ car elle est dans la composante connexe de l'élément neutre du centralisateur de $L \subset K$. Par suite, cette composante connexe du centre de $K$ est compacte. Puisque $K$ est connexe (10) et localement isomorphe au groupe compact $K_{a}$, il en résulte que $K$ est compact. Ainsi, le centre $Z$ de $G$ est un sousgroupe fini de $G$ et les sous-groupes compacts maximaux de $G$ et de $G_{a}$ ont même dimension.

Soit $\mathfrak{l}$ la sous-algèbre des $X \in \mathfrak{g}$ qui, au point neutre, sont tangents au sousgroupe compact maximal $K$ de $G$. Puisque $\mathfrak{l}$ est également la sous-algèbre qui correspond au sous-groupe compact maximal $K_{a}$ de $G_{a}$, il existe un automorphisme involutif $\sigma$ de l'algèbre de Lie $\mathfrak{g}$ tel que $\mathfrak{l}$ soit la sous-algèbre des éléments invariante par $\sigma$. De plus, les éléments de l'algèbre de Lie g ${ }^{C}$ complexifiée de $\mathfrak{g}$ qui sont de la forme $X+i Y$ avec $\sigma . X=X$ et $\sigma . Y=-Y$ constituent une sous-algèbre de Lie $\mathfrak{g}^{\prime}$ (sur les nombres réels) qui est une forme compacte (4) de $\mathfrak{g}$. Soit $\mathfrak{h}$ une sous-algèbre abélienne maximale de $\mathfrak{g}$ contenant la sous-algèbre de dimension 1 qui correspond à $L$. On a $\mathfrak{h} \subset \mathfrak{b}$ et $\mathfrak{h}$ est également une sous-algèbre abélienne maximale de $\mathfrak{g}^{\prime}$. Par suite, la sous-algèbre complexe $\mathfrak{h}^{C}$ de $\mathfrak{g}^{C}$ engendrée par $\mathfrak{h}$ est une sous-algèbre de Cartan de $\mathfrak{g}^{C}$. Soient $X_{\gamma}$ des vecteurs propres non nuls correspondant aux racines $\gamma \neq 0$ de $\mathfrak{g}^{C}$ relatives à cette sous-algèbre de Cartan. Si $\sigma^{C}$ désigne le prolongement de $\sigma$ en automorphisme complexe de $\mathfrak{g}^{C}$, on a $\sigma^{C} . H=H$ pour tout $H \in \mathfrak{h}^{C}$ et par suite $\sigma^{C} . X_{\gamma}= \pm X_{\gamma}$ pour toute racine $\gamma$.

Soit $G^{\prime}$ le groupe semi-simple compact adjoint de $\mathfrak{g}^{\prime}$. Le sous-groupe compact $K_{a}$ de $G_{a}$ s'identifie à un sous-groupe de $G^{\prime}$ et, dans cette identification, $B_{a}$ devient le sous-groupe fermé de $G^{\prime}$ qui correspond à la sous-algèbre $\mathfrak{b} \subset \mathfrak{g}^{\prime}$. Ce sous-groupe $B_{a}$ de $G^{\prime}$ est le centralisateur dans $G^{\prime}$ du sous-groupe image de $L$ dans $B / Z=B_{a} \subset G^{\prime}$. Par conséquent, l'espace homogène $G^{\prime} / B_{a}$ possède des structures complexes invariantes en nombre fini (13). Soit $J^{\prime}$ un endomorphisme de l'espace $\mathrm{g}^{\prime}$ définissant une de ces structures complexes et soit $J^{C}$ son prolongement en endomorphisme complexe de l'espace $\mathrm{g}^{C}$. Des conditions $(2.2),(2.3),(2.4)$, et (2.6) vérifiées par $J^{\prime}$, on déduit que $J^{C}$ vérifie les conditions

$$
\begin{aligned}
& J^{C} \cdot \mathfrak{b}^{C}=(0) \\
& \left(J^{C}\right)^{2} \cdot X=-X\left(\bmod \mathfrak{b}^{C}\right) \text { pour tout } X \in \mathfrak{g}^{C} \\
& J^{C} \cdot[X, Y]=\left[X, J^{C} . Y\right]\left(\bmod \mathfrak{b}^{C}\right) \text { quels que soient } X \in \mathfrak{b}^{C} \text { et } Y \in \mathfrak{g}^{C}, \\
& {[X, Y]+J^{C} \cdot\left[J^{C} . X, Y\right]+J^{C} \cdot\left[X, J^{C} . Y\right]-\left[J^{C} . X, J^{C} . Y\right] \in \mathfrak{b}^{C}} \\
& \text { quels que soient } X, Y \in \mathfrak{g}^{C}
\end{aligned}
$$

Il résulte de (6.3) que, pour toute racine $\gamma \neq 0$,

$$
J^{C} . X_{\gamma}= \pm i X_{\gamma}\left(\bmod \mathfrak{b}^{C}\right) \text {. }
$$

Par suite, $J^{C} \sigma^{C}-\sigma^{C} J^{C}$ applique $\mathfrak{g}^{C}$ dans $\mathfrak{b}^{C}$ et par conséquent, $J^{C} \cdot \mathfrak{g} \subset \mathfrak{g}$. La restriction de $J^{C}$ à $\mathfrak{g}$ est un endomorphisme $J$ de l'espace $\mathfrak{g}$ qui vérifie 
visiblement les conditions (2.2), (2.3) et (2.6). Il vérifie aussi la condition (2.4) car, $B$ étant connexe, cette condition est équivalente à la condition $J .[X, Y]=[X, J . Y](\bmod \mathfrak{b})$ quels que soient $X \in \mathfrak{b}, Y \in \mathfrak{g}$, laquelle résulte de (6.3). Ainsi, à toute structure complexe invariante sur $G^{\prime} / B_{a}$ est associée une structure complexe invariante sur $G / B$. Un raisonnement analogue montre que. réciproquement, toute structure complexe invariante sur $G / B$ est ainsi associée à une structure complexe invariante sur $G^{\prime} / B_{a}$

Partant d'une structure complexe invariante sur $G^{\prime} / B_{a}$ définie par $J^{\prime}$ et munissant $G / B$ de la structure complexe invariante associée, nous allons comparer les formes hermitiennes canoniques de $G^{\prime} / B_{a}$ et de $G / B$. D'après (6.3), quels que soit $X \in \mathrm{g}^{C}$, l'endomorphisme $\operatorname{ad}\left(J^{C} . X\right)-J^{C} \operatorname{ad}(X)$ laisse stable $\mathfrak{b}^{C}$. La trace de l'endomorphisme complexe de $\mathfrak{g}^{C} / \mathfrak{b}^{C}$ déduit de ad $\left(J^{C} . X\right)$ $-J^{C}$ ad $(X)$ par passage au quotient est une fonction linéaire complexe de $X \in \mathfrak{g}^{C}$ que l'on note $\psi^{C}(X)$. Les formes $\psi$ et $\psi^{\prime}$ pour $G / B$ et $G^{\prime} / B_{a}$ sont respectivement égales aux restrictions de $\psi^{C}$ à $\mathfrak{g}$ et à $\mathfrak{g}^{\prime}$. Compte tenu de (4.2) et (4.6), on voit donc que les formes $\eta$ et $\eta^{\prime}$, images inverses des formes hermitiennes canoniques de $G / B$ et $G^{\prime} / B$, sont égales respectivement aux restrictions à $\mathfrak{g}$ et $\mathfrak{g}^{\prime}$ de la forme bilinéaire complexe

$$
\eta^{C}(X, Y)=\frac{1}{2} \psi^{C}\left(\left[J^{C} . Y, Y\right]\right) .
$$

Comme $\sigma^{C}$ est un automorphisme de $\mathfrak{g}$ et que $\sigma^{C} J^{C}-J^{C} \sigma^{C}$ applique $\mathfrak{g}^{C}$ dans $\mathfrak{b}^{C}$, on voit que $\psi^{C}\left(\sigma^{C} . X\right)=\psi^{C}(X)$ pour tout $X \in \mathfrak{g}^{C}$. La forme $\eta^{C}$ est donc invariante par $\sigma^{C}$; en particulier, si $\sigma^{C} . X=X$ et $\sigma^{C} . Y=-Y$, on a $\eta^{C}(X, Y)=0$. On en déduit que

(6.5) L'image inverse $\eta$ de la forme canonique de $G / B$ se déduit de l'image inverse $\eta_{\mathbf{k}}^{\prime}$ de la forme canonique de $G^{\prime} / B_{a}$ par les relations:

$$
\begin{array}{ll}
\eta(X, Y)=\eta^{\prime}(X, Y) & \text { si } \sigma . X=X \text { et } \sigma . Y=Y, \\
\eta(X, Y)=0 & \text { si } \sigma . X=X \text { et } \sigma . Y=-Y, \\
\eta(X, Y)=-\eta^{\prime}(i X, i Y) & \text { si } \sigma . X=-X \text { et } \sigma . Y=-Y .
\end{array}
$$

On est ainsi ramené à l'étude du cas où $G$ est compact, $\mathfrak{g}^{\prime}$ étant donc l'algèbre de Lie $\mathfrak{g}$ elle-même. Puisque $\mathfrak{g}$ est une forme compacte de $\mathfrak{g}^{C}$, toute racine $\alpha$ relative à $\mathfrak{h}^{C}$ vérifie $\bar{\alpha}=-\alpha$. Pour toute racine $\alpha \neq 0$, soit $H_{\alpha}^{\prime}$ l'élément de $\mathfrak{h}^{C}$ tel que $\alpha(H)=\operatorname{Tr}$ ad $\left(H_{\alpha}{ }^{\prime}\right)$ ad $(H)$ pour tout $H \in \mathfrak{h}^{C}$. On sait (14) que les $X_{\alpha}$ peuvent être choisis de telle sorte que, pour toute racine $\alpha \neq 0$,

$$
\bar{X}_{\alpha}=-X_{-\alpha}, \quad\left[X_{\alpha}, X_{-\alpha}\right]=H_{\alpha} \text { où } H_{\alpha}=2 \alpha\left(H_{\alpha}^{\prime}\right)^{-1} H_{\alpha}^{\prime}
$$

Le sous-groupe $B$ étant compact, on peut supposer que l'endomorphisme $J$ de $\mathfrak{g}$ qui définit la structure complexe invariante sur $G / B$ commute avec $\operatorname{ad}(X)$ pour tout $X \in \mathfrak{b}$. Les $X_{\alpha}$ sont alors des vecteurs propres appartenant aux racines $0, i$ ou $-i$ de $J^{c}$. Soit $\mathscr{D}$ l'ensemble des racines $\alpha \neq 0$ telles que $J^{C} . X=i X$; les $X_{\alpha}$ et $X_{-\alpha}$ pour $\alpha \in \mathscr{D}$ constituent une base d'un supplémentaire complexe de $\mathfrak{b}^{C}$ dans $\mathfrak{g}^{C}$. Pour toute racine $\gamma \neq 0$, on a $X_{\gamma} \in\left[\mathfrak{b}^{C}, \mathfrak{g}^{C}\right]$ 
et par suite $\psi^{C}\left(X_{\gamma}\right)=0$. Pour tout $H \in \mathfrak{h}^{C}$, on a $J^{C} . H=0$ et $J^{C} \operatorname{ad}(H) . X_{\alpha}=i \alpha(H) X_{\alpha}$ lorsque $\alpha \in \mathscr{D}$. La définition de $\psi^{C}$ donne donc ${ }^{9}$

$$
\psi^{C}(H)=-2 i \sum \alpha(H)
$$

$\alpha \in \mathscr{D}$.

Les $U_{\alpha}=X_{\alpha}-X_{-\alpha}$ et $V_{\alpha}=i\left(X_{\alpha}+X_{-\alpha}\right)$ pour $\alpha \in \mathscr{D}$ constituent une base d'un supplémentaire de $\mathfrak{b}$ dans g. On a $J \cdot U_{\alpha}=V_{\alpha}$ et $J . V_{\alpha}=-U_{\alpha}$ pour tout $\alpha \in \mathscr{D}$. Si $\alpha$ et $\beta$ sont des racines distinctes, $\left[U_{\alpha}, V_{\beta}\right]$ est combinaison linéaire des $X_{\gamma}$ appartenant aux racines $\gamma \neq 0$; il en est de même de $\left[U_{\alpha}, U_{\beta}\right]$ et $\left[V_{\alpha}, V_{\beta}\right]$ quelles que soient les racines $\alpha, \beta \neq 0$. Puisque $\psi^{C}\left(X_{\gamma}\right)=0$ pour $\gamma \neq 0$, il résulte de (4.6) que les $U_{\alpha}$ et $V_{\beta}$ sont deux à deux orthogonaux pour la forme $\eta$. Compte tenu de (6.6), on a

$$
\eta\left(U_{\alpha}, U_{\alpha}\right)=\eta\left(V_{\alpha}, V_{\alpha}\right)=\frac{1}{2} \psi\left(\left[J . U_{\alpha}, U_{\alpha}\right]\right)=-\frac{1}{2} i \psi^{c}\left(H_{\alpha}\right)
$$

pour toute racine $\alpha \neq 0$.

Lemme 2. Pour toute racine $\gamma \in \mathscr{D}$, on a $\sum \alpha\left(H_{\gamma}\right)>0$ et pour toute racine $\gamma \neq 0$ telle que $X_{\gamma} \in \mathfrak{b}^{C}$, on a $\sum \alpha\left(H_{\gamma}\right)=0$, les sommes portant sur toutes les racines $\alpha \in \mathscr{D}$.

Soit $\mathscr{G}$ l'ensemble de racines comportant la racine 0 , les racines dans $\mathscr{D}$ et les racines $\alpha \neq 0$ telles que $X_{\alpha} \in \mathfrak{b}^{C}$. Pour qu'une racine $\alpha$ soit dans $\mathscr{G}-\mathscr{D}$, il faut et il suffit que $\alpha \in \mathscr{G}$ et $-\alpha \in \mathscr{G}$. La condition d'intégrabilité (2.6) vérifiée par $J$ signifie que le sous-espace complexe engendré par les vecteurs. propres qui appartiennent à une racine dans $\mathscr{G}$ est une sous-algèbre de Lie de $\mathrm{g}^{c}$. Par suite, si $\alpha, \beta \in \mathscr{G}$ et si $\alpha+\beta$ est racine, alors $\alpha+\beta \in \mathscr{G}$. Il en résulte que, si $\alpha \in \mathscr{G}, \beta \in \mathscr{D}$ et si $\alpha+\beta$ est racine, alors $\alpha+\beta \in \mathscr{D}$. On a en effet $\alpha+\beta \in \mathscr{G}$ et si $\alpha+\beta$ était dans $\mathscr{G}-\mathscr{D}$, on aurait $-(\alpha+\beta) \in \mathscr{G}$, donc $-\beta=\alpha-(\alpha+\beta) \in \mathscr{G}$, d'où $\beta \in \mathscr{G}-\mathscr{D}$ contrairement à l'hypothèse. Soit $\gamma$ une racine $\neq 0$ dans $\mathscr{G}$ et soit $\beta$ une racine telle que $\beta+\gamma$ ne soit pas racine. On appellera classe de $\beta$ (relativement à $\gamma$ ) l'ensemble $\mathscr{D}_{\beta}$ des racines: dans $\mathscr{D}$ qui sont de la forme $\beta+k \gamma$ ( $k$ entier). Comme l'ensemble de toutes les racines de la forme $\beta+k \gamma$ s'obtient pour $p \leqslant k \leqslant 0$ où $p$ est un entier $\leqslant 0$, il résulte de la remarque précédente que, si $\mathscr{D}_{\beta} \neq \phi$, il existe un entier $s$ vérifiant $p \leqslant s \leqslant 0$ tel que $\mathscr{D}_{\beta}$ soit l'ensemble des racines de la forme $\beta+k \gamma$ avec $s \leqslant k \leqslant 0$. Si $\beta$ et $\gamma$ sont linéairement indépendantes, on sait (14) que $\beta\left(H_{\gamma}\right)=-p$. Puisque $\gamma\left(H_{\gamma}\right)=2$, on a donc dans ce cas

$$
\begin{aligned}
\sum \alpha\left(H_{\gamma}\right) & =(\beta+\beta-\gamma+\ldots+\beta+s \gamma)\left(H_{\gamma}\right) \\
& =(1-s)(s-p) \geqslant 0 .
\end{aligned}
$$

Si $\beta$ et $\gamma$ sont linéairement dépendantes, alors $\beta=\gamma$, car $\beta=0$ et $\beta=-\gamma$ sont exclus du fait que $\beta+\gamma$ n'est pas racine. Dans ce cas, si $\mathscr{D}_{\beta} \neq \phi$, c'est donc que $\gamma \in \mathscr{D}$ et $\mathscr{D}_{\beta}=(\gamma)$, donc $\sum \alpha\left(H_{\gamma}\right)=2>0$ pour $\alpha \in \mathscr{D}_{\beta}$. Comme

\footnotetext{
${ }^{9} \mathrm{La}$ restriction de $\psi^{C}$ à $\mathfrak{h}$ est bien une forme réelle car, si $H=\bar{H}$, on a $\overline{\alpha(H)}=-\alpha(H)$ pour toute $\alpha$.
} 
toute racine dans $\mathscr{D}$ appartient à une classe et une seule, on peut calculer $\sum \alpha\left(H_{\gamma}\right)$ pour $\alpha \in \mathscr{D}$, en groupant les racines $\alpha$ par classes. Si $\gamma \in \mathscr{D}$, les sommes partielles sont toutes $\geqslant 0$ et la somme partielle relative à $\mathscr{D}_{\gamma}$ est $>0$, d'où la première partie du Lemme. Si $\gamma \in \mathscr{G}-\mathscr{D}$, alors $-\gamma \in \mathscr{G}$; pour toute racine $\beta$ telle que $\mathscr{D}_{\beta} \neq 0, \gamma$ est linéairement indépendante de $\beta$ et $p=s$. On a donc $\sum \alpha\left(H_{\gamma}\right)=0$ pour $\alpha \in \mathscr{D}_{\beta}$, d'où la seconde partie du Lemme ${ }^{10}$.

De ce Lemme et des formules (6.7), (6.8), il résulte que la forme hermitienne canonique de $G / B$ est définie négative. Compte tenu des relations (6.5) qui ramènent le cas d'un groupe $G$ semi-simple au cas où $G$ est compact, on obtient les Théorèmes suivants:

ThÉORغ̀me 2. Soit $G$ un groupe semi-simple et soit $L$ un sous-groupe à un paramètre de G. Si un sous-groupe compact $B$ de $G$ est un sous-groupe ouvert $d u$ centralisateur de $L$, alors $B$ est connexe et l'espace homogène $G / B$ possède des structures complexes invariantes. Pour chacune de ces structures la forme hermitienne canonique de $G / B$ est non dégénérée. Le nombre de ses carrés négatifs est égal à la différence entre la dimension des sous-groupes compacts maximaux de $G$ et la dimension de $B$.

ThÉorème 3. Si $G$ est un groupe compact et si $G / B$ est un espace homogène complexe dont la forme hermitienne canonique est non dégénérée, alors cette forme est définie négative.

En effet, la composante connexe de l'élément neutre dans le centre de $G$ opère trivialement dans $G / B$ d'après les résultats du $\S 5$. On peut donc supposer $G$ semi-simple, et la remarque (5.2) permet d'appliquer ${ }^{11}$ le Théorème 2 .

THÉorÈme 4. Soient $G$ un groupe semi-simple et $B$ un sous-groupe compact de $G$. S'il existe sur l'espace homogène $G / B$ une structure complexe invariante pour laquelle la forme hermitienne canonique est non dégénérée, alors $B$ est connexe, le centre de $G$ est fini et le nombre des carrés négatifs de la forme canonique est égal à la différence entre la dimension des sous-groupes compacts maximaux de $G$ et la dimension de $B$.

C'est encore une conséquence de la remarque (5.2) et du Théorème 2. En particulier, il résulte du Théorème 4 que si la forme hermitienne canonique

\footnotetext{
${ }^{10}$ Nous n'utiliserons ici que la premiere partie du Lemme. La seconde a l'intéret de montrer que les racines $\gamma \leqslant \mathscr{D}$ peuvent être caractérisées par une inégalité de la forme $\lambda\left(H_{\gamma}\right) \leqslant 0$ où $\lambda$ est une fonction linéaire sur $\mathfrak{h} c$. On obtient ainsi une manière de déterminer toutes les structures complexes invariantes sur $G / B(\mathbf{3} ; \mathbf{1 3})$.

${ }^{11}$ D'après un Théorème de Montgomery (Simply connected homogeneous spaces, Proc. Amer. Math. Soc., 1 (1950), 467-469), si $G / B$ est compact et a un groupe de Poincaré fini, tout sous-groupe compact maximal dans $G$ opère transitivement sur $G / B$. Par suite, si un espace homogène complexe compact a un groupe de Poincaré fini et si sa forme hermitienne canonique est non dégénérée, alors cette forme est définie négative. Les Théorèmes 3 et 4 sont en rapports étroits avec les résultats de Bochner et Lichnerowicz sur les champs de vecteurs de Killing et le tenseur de Ricci $(2 ; \mathbf{1 2})$.
} 
est définie positive, alors $B$ est un sous-groupe compact maximal et $G / B$ est un espace riemannien symétrique. Ceci montre que les domaines bornés homogènes dont le groupe des automorphismes est semi-simple sont des domaines bornés symétriques au sens de E. Cartan (5); on sait en effet que leur forme hermitienne canonique est définie positive (1) et que le groupe de stabilité d'un point est compact (6). Une démonstration plus directe de ce résultat a été donnée par A. Borel qui suppose seulement que le groupe de tous les automorphismes du domaine borné contient un sous-groupe transitif semisimple $(3)^{12}$.

${ }^{12} \mathrm{On}$ peut conjecturer que la non dégénérescence de la forme hermitienne canonique d'un espace homogène complexe $G / B$ correspond à une propriété intrinsèque du plus grand groupe des automorphismes analytiques de $G / B$ qui conservent le volume (peut être sa semi-simplicité). D'une manière générale, il serait utile de savoir jusqu'où va l'analogie que nos résultats semblent indiquer entre la forme de Killing d'un groupe et la forme hermitienne canonique d'un espace homogène complexe.

\section{BiLLIOGRAPHIE}

1. S. Bergman, Über die Kernfunktion eines Bereiches und ihr Verhalten am Rande, J. reine und angew. Math., 169 (1932), 1-42.

2. S. Bochner, Laplace operator on manifolds. Int. Congress of Math. (Cambridge, 1950), 189-201.

3. A. Borel, Kaehlerian coset spaces of semi-simple Lie groups, Proc. Nat. Acad. Sci., 40 (1954), 1147-1151.

4. E. Cartan, Groupes simples clos et ouverts et géométrie riemannienne, J. Math. pures et appliquées, 8 (1929), 1-33.

5. - - Sur les domaines bornés de l'espace de $n$ variables complexes, Abh. Math. Seminar Hamburg, 11 (1935), 116-162.

6. H. Cartan, Sur les groupes de transformations analytiques (Paris, 1935).

7. - Notions d'algèbre différentielle . . . , Coll. de Topologie, Bruxelles (1950), 15-27.

8. B. Eckmann et A. Frölicher, Sur l'intégrabilité des structures presque complexes, C. R. Acad. Sci. Paris, 232 (1951), 2284-2286.

9. H. Hopf, Über den Rang geschlossener Liescher Gruppen, Comm. Math. Helv., 19 (1940), 119-155.

10. K. Iwasawa, On some types of topological groups, Ann. Math., 50 (1949), 507-558.

11. J. L. Koszul, Homologie et cohomologie des algèbres de Lie, Bull. Soc. Math. France, 78 (1950), 65-127.

12. A. Lichnerowicz, Espaces homogènes Kaehleriens, Coll. Géom Diff. (Strasbourg, 1953), 171-184.

13. H. C. Wang, Closed manifolds with homogeneous complex structures, Amer. J. Math., 76 (1954), 1-32.

14. H. Weyl, Theorie der Darstellung kontinuerlicher halb-einfach Gruppen durch lineare Transformationen, Math. Z., 23 (1925), 271-309; 24 (1926), 238-395.

\section{Université de Strasbourg}

